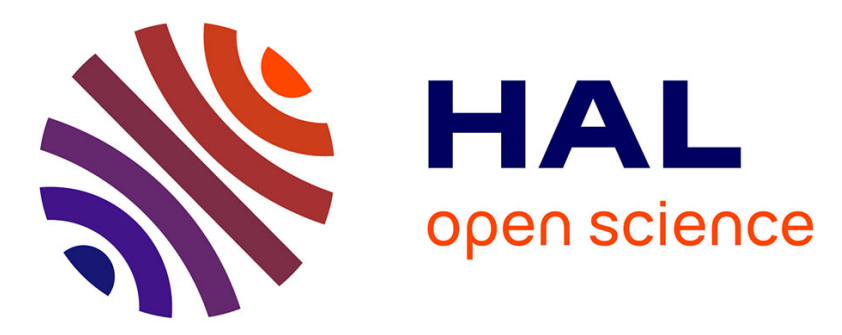

\title{
Design and Optimization of a Multi-drone Robot for Grasping and Manipulation of Large-size Objects
}

Marie Saint-Sevin, Vincent Begoc, Sébastien Briot, Abdelhamid Chriette, Isabelle Fantoni

\section{- To cite this version:}

Marie Saint-Sevin, Vincent Begoc, Sébastien Briot, Abdelhamid Chriette, Isabelle Fantoni. Design and Optimization of a Multi-drone Robot for Grasping and Manipulation of Large-size Objects. 22nd CISM IFToMM Symposium on Robot Design, Dynamics and Control (RoManSy 2018), Jun 2018, Rennes, France. 10.1007/978-3-319-78963-7_57 . hal-01759340v2

HAL Id: hal-01759340

https://hal.science/hal-01759340v2

Submitted on 23 Sep 2019

HAL is a multi-disciplinary open access archive for the deposit and dissemination of scientific research documents, whether they are published or not. The documents may come from teaching and research institutions in France or abroad, or from public or private research centers.
L'archive ouverte pluridisciplinaire HAL, est destinée au dépôt et à la diffusion de documents scientifiques de niveau recherche, publiés ou non, émanant des établissements d'enseignement et de recherche français ou étrangers, des laboratoires publics ou privés. 


\title{
Design and Optimization of a Multi-drone Robot for Grasping and Manipulation of Large size Objects
}

\author{
Marie Saint-Sevin ${ }^{1}$, Vincent Bégoc ${ }^{1,4}$, Sébastien Briot ${ }^{2,4}$, Abdelhamid \\ Chriette $^{3,4}$, and Isabelle Fantoni ${ }^{2,4}$ \\ 1 Institut Catholique d'Arts et Métiers (Icam), France \\ vincent.begoc@icam.fr, \\ 2 Centre National de la Recherche Scientifique (CNRS), France \\ 3 École Centrale Nantes, France \\ 4 Laboratoire des Sciences du Numérique de Nantes (LS2N), UMR CNRS 6004, \\ Nantes, France
}

\begin{abstract}
This paper presents a new type of flying robot dedicated to grasping and manipulation of large size objects. The system can be basically presented as an aerial hand with four fingers actuated by four quadrotors whose arrangement permits the manipulation of the grasped object in the space. Each finger has two phalanges and is underactuated in order to adapt itself to the object size and shape. The opening/closing motion of each finger is actuated by the yaw motion of each quadrotor and transmitted through a non-backdrivable mechanism in order to enable the system to produce form-closed grasps. This stability criteria yields to secured grasps which do not rely on the capability of actuators nor on the contact friction between the phalanges and the object, and furthermore do not require any additional energy for gripping during the flight. The present paper gives guidelines to optimize the geometric parameters of a planar aerial robot in order to maximize the robot's manipulability and its capability to produce form-closed grasps.
\end{abstract}

Keywords: Flying robot, grasping, manipulation, underactuated system, optimization, manipulability

\section{Introduction}

Researchers communities have shown an increasing interest in new uses of Unmanned Aerial Vehicles (UAVs), especially for interactions with the environment. Recent works have been devoted to drones able to grasp objects: $[14,9,11,10]$ introduced UAVs under which a gripper was mounted. A drawback of this kind of solution is that it allows for very low dexterity during the manipulation process.

Further works explored solutions where a serial manipulator, equipped with a gripper, is attached to a UAV $[13,4]$. However, such robots present a low payload and a reduced energy autonomy due to respectively the weight and the energy consumption of the embedded serial manipulator. 


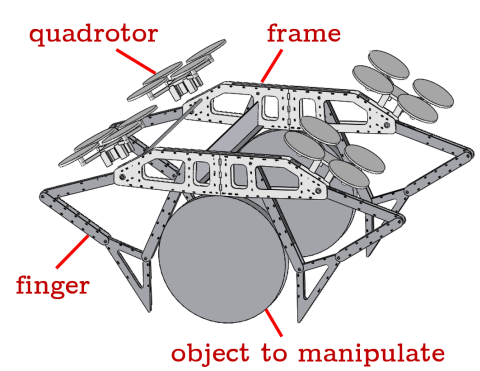

(a) Global view of the manipulator

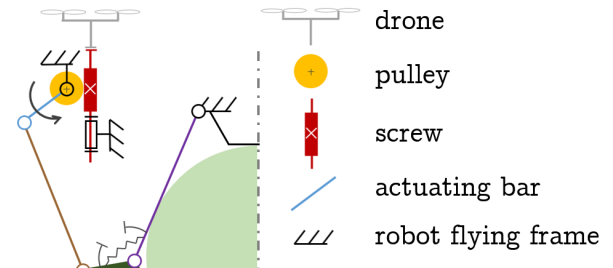

(b) Kinematic chain of each finger

Fig. 1: Flying manipulator presentation

To overcome those limitations, other works have explored the collaboration of several drones associated with cables $[3,8]$ or with rigid bodies $[7,12]$. However, these solutions have been designed for manipulation tasks and not for grasping.

[2] proposed a system named "the flying hand" which is basically a fleet of drones coming into contact with an object in order to manipulate it, each drone being considered as a finger. However, this system can not ensure the security of the grasp since it requires a very precise and robust control of each drone to continuously exert a force by each finger on the object, and the stability depends on contact friction between fingers and the object which can change with humidity for example.

In the present paper, we introduce a new aerial mechanism devoted to grasping and manipulation. This mechanism is basically an aerial hand in which the motors for closing the fingers have been replaced by drones. Fingers are underactuated so that it can automatically adapt to the object shape and size without adding actuators which burden the structure, decrease energetic autonomy and require complex control algorithms [1]. Our new flying manipulator ensures grasp stability, thanks to the use of non-backdrivable mechanisms which permit to achieve form-closure grasps [5]. Form-closure does not rely on friction forces and thus, requires no energy for the gripping task during the flight. In what follows, we introduce the robot architecture and its optimal design.

\section{Presentation of the robot architecture}

The robot is composed of a frame, four quadrotors and four two-phalanx planar fingers. In the proposed solution, all finger plane-of-motions are parallel (Fig. 1a). A four-bar mechanism (Fig. 2) is used to underactuate the fingers so that they can automatically adapt to the shape and size of the object and be less sensitive to positioning errors of the robot relative to the object to grasp. In Fig. 2(a), the finger is in its initial configuration and behaves as a rigid body. The bar (1) rotates until the proximal phalanx (3) comes into contact with the object 


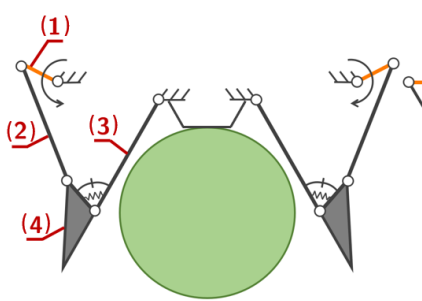

(a)

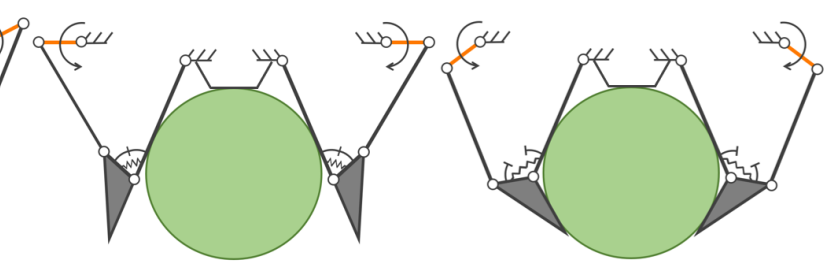

(b)

(c)

Fig. 2: Closing sequence of a two-phalanx finger with a four-bar linkage transmission [1] (actuation bar in orange).

(Fig. 2(b)). The second phalanx (4) begins its rotation as soon as the torque produced by the actuation bar gets larger than the torque exerted by the spring, until it finally reaches the object (Fig. 2(c)).

The opening/closing motion of each finger is driven by the bar (1) which is actuated by a quadrotor linked to the frame by a revolute joint (Fig. 1b). This degree of freedom enables the drone to use its yaw motion to drive a nonbackdrivable worm-gear mechanism whose pulley is fixed to the actuation bar of the finger. As mentionned in [5], a two-finger planar gripper requires at least two unilateral mechanisms to produce 1st-order form-closed grasps of a cylindrical object in the plane (rotation of the object is not considered). Worm-gears are chosen with opposite hand (left and right) so that drones must turn in the opposite direction to close fingers during the grasping process. The resulting torque exerted on the frame along the vertical axis is null.

Since a quadrotor is underactuated (four propellers operate in a six-dimensional space), we introduce a tilting angle between both quadrotors in order to obtain a fully actuated mechanism in the plane. This allows the full manipulation of the grasped object in a vertical plane (Fig. 3a).

\section{Optimization of the manipulability}

In this section, we consider a planar manipulator actuated by two drones as presented in Fig. 3a and we optimize the position and orientation of drones with respect to the frame in order to maximize its capacity to manipulate an object in the plane. The aim of this study is to ensure that drones are able to exert forces on the frame along both directions of the plane. In our case, rotational motion is not needed, however, the manipulator must resist to disturbance torque about the orthogonal axis. The grasped object is supposed to be fixed relatively to the frame.

In [6], the author introduces a force performance index: the maximum isotropic operational force, namely the maximal force the manipulator can exert in any direction of its operational space. It consists in computing the radius of the largest circle included in the convex hull of admissible operational forces. This latter is 


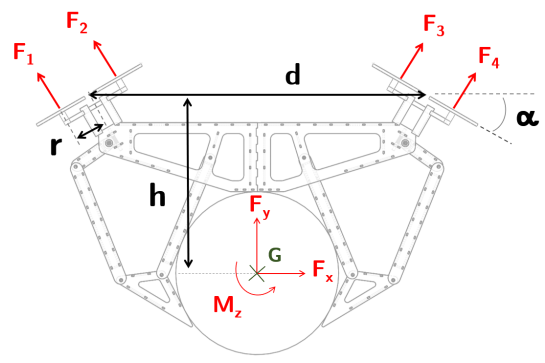

(a) Geometric parameters and forces of the manipulator

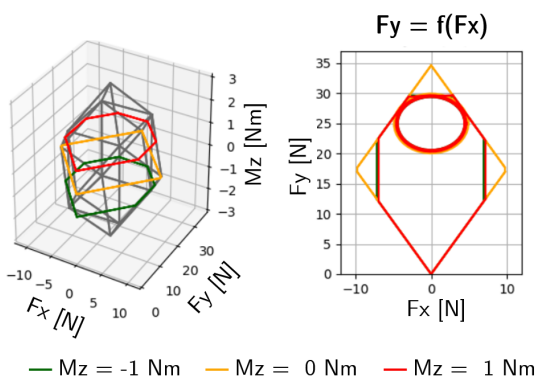

(b) Admissible operational force space for the optimal case

Fig. 3: Manipulability study

obtained by mapping the admissible actuator space in the operational space by using the expressions given in [6].

The admissible actuator space represents all realistic combinations of actuator forces. For the sake of simplicity, the four propellers of a quadrotor are modeled with two forces. The admissible actuator space is thus a 4-dimensional cube whose boundaries are defined by $F_{\min }<F_{i}<F_{\max }, i \in\{1, \ldots, 4\}$ derived from the propellers capabilities. Considering that each propeller can deliver a maximal thrust force of $5 \mathrm{~N}$, we obtain $0<F_{i}<10 \mathrm{~N}$, the admissible operational force convex hull represented in Fig. $3 \mathrm{~b}$ can thus be obtained by using the expressions given in [6].

Our force performance index is $r$, the radius of the largest circle included in the three polygons defined by the intersection of the operational force space with the three planes defined by $M_{z}=-1 \mathrm{Nm}, M_{z}=0 \mathrm{Nm}$ and $M_{z}=1 \mathrm{Nm}$ (chosen as limit values for the maximal accepted disturbance torque; see in Fig. $3 \mathrm{~b}$ the convex hull obtained when using parameters of Tab. 1). Taking into account the gravity field of norm $g$, the circle is centered on $\left(F_{x}, F_{y}\right)=(0, m g)$, with $m=2.5 \mathrm{~kg}$ the weight of the system \{quadrotors + gripper + object $\}$.

The geometrical parameters of the manipulator's frame are $\alpha$ (characterizing the orientation of the propellers), $h$ (the distance to the object center) and $d$ (the distance between the two quadrotors) (Fig. 3a). $\alpha$ and $h$ are the parameters to optimize. $d=770 \mathrm{~mm}$ is an input parameter set to, what authors consider, the minimal distance between the quadrotors in order to avoid aerodynamic disturbance.

The optimization process consists in varying $\alpha$ and $h$ in a large interval (with small increments for high precision). For each couple of parameters, the performance index is calculated and we thus obtain the graphic presented in Fig. 4. Note that the operational forces are calculated at the object gravity center $G$, for a $300 \mathrm{~mm}$ radius cylindrical object. The best couple of design parameters based on the study of the Fig. 4 is specified in Tab. 1, with minimal $h$ so as to reduce the mass of the manipulator. It leads to a force capacity of $F_{x}=F_{y}= \pm 4.84 \mathrm{~N}$ for manipulating the aerial robot. 


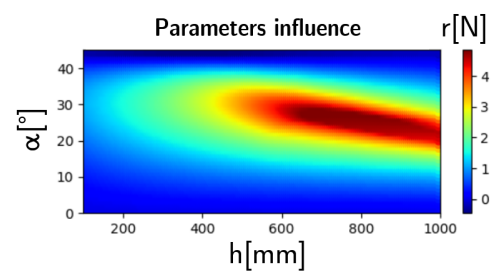

Fig. 4: Graphic resulting from the manipulability optimization

\begin{tabular}{c|c|c|c|c}
\multicolumn{2}{c|}{ Input parameters } & \multicolumn{2}{c}{ Results } \\
\hline$r[\mathrm{~mm}]$ & $m[\mathrm{~kg}]$ & $d[\mathrm{~mm}]$ & $\left.\alpha{ }^{\circ}\right]$ & $h[\mathrm{~mm}]$ \\
\hline 170 & 2.5 & 770 & 28 & 660
\end{tabular}

Table 1: Optimization for manipulability: parameters and results

\section{Form-closure optimization}

Even if underactuated hands permit to produce enveloping grasp that should be, a priori, more robust than a two-contact grasp, in some configurations, such underactuated hands exhibit aberrant behavior leading to the ejection of the object [1]. To avoid this phenomenon, it is necessary to optimize the geometry of the gripper at the design stage.

In [5], the authors present a method to analyze the form-closure of an underactuated grasp. This type of stability is a purely geometrical property and thus, does not depend on actuators capability, friction forces, nor on the magnitude of external disturbances. Form-closure is thus an ideal property to attain with a flying gripper since it guarantees that the object will not fall.

\subsection{Form-closure analysis}

In [5], form-closure is reformulated for underactuated grasps as follows: "A grasp is said to be form-closed if, and only if, for any variation of the configuration of the grasp at least one of the unilateral kinematic constraints is violated." A 1st-order modeling of the relations between the configuration of the grasp and the unilateral kinematic constraints is given by matrix $\mathbf{M}$ defined as:

$$
\dot{\mathbf{q}}_{\mathbf{c}}=\mathbf{M} \dot{\mathbf{w}} \text { where } \mathbf{M}=\left[\begin{array}{cc}
\mathbf{P} & -\mathbf{J} \\
\mathbf{0} & \mathbf{K} \\
0 & 1
\end{array}\right]
$$

with:

- $\dot{\mathbf{q}}_{\mathbf{c}}$ is the unilateral constraints vector, including the contact constraints (between the object, the four phalanges and the palm) and the unilateral constraints imposed by worm-gear mechanisms. Each component of this vector has to be positive or null otherwise the corresponding constraint is violated.

- $\dot{\mathbf{w}}=\left[\dot{\mathbf{u}}^{T}, \dot{\boldsymbol{\theta}}^{T}\right]^{T}$ with $\dot{\mathbf{u}}$ the vector of operational velocities of the object and $\dot{\boldsymbol{\theta}}$ the vector of joint velocities of the hand,

- $\mathbf{P}$ is the projection matrix, $\mathbf{J}$ the hand Jacobian matrix and $\mathbf{K}$ the unidirectional mechanisms constraints matrix. Their expressions are given in [5]. 


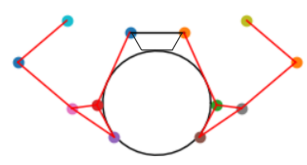

(a) $\delta_{x}=0 \mathrm{~mm}$, form-closed

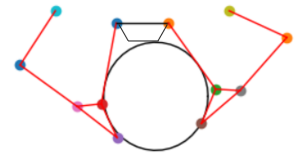

(b) $\delta_{x}=83 \mathrm{~mm}$, not form-closed

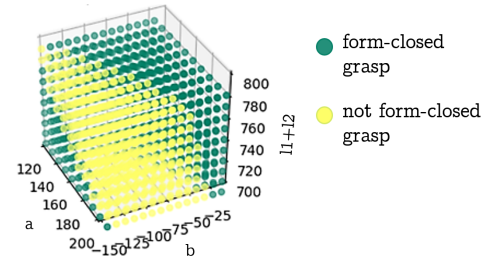

(c) Influence of 3 geometric parameters: $a, b, l_{1}+l_{2}$

Fig. 5: Form-closure evolution.

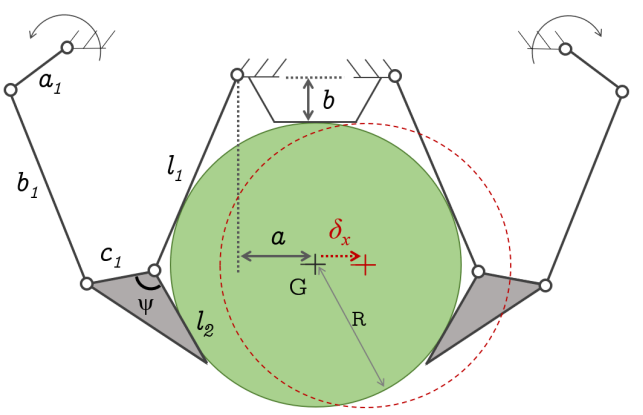

Fig. 6: Model of the manipulator parameters

The method presented in [5] is based on the study of the convex hull of matrix $\mathbf{M}$. A grasp is first-order form-closed if and only if $\operatorname{conv}(\mathbf{M})$ strictly contains the origin of the configuration space of the grasp. Using this method shows us that form-closure depends on the position of the grasped object as depicted in Fig. 5a and Fig. 5b. In the same manner, form-closure also depends on the geometric parameters of the fingers depicted in Fig. 5c.

\subsection{Optimization method}

Since form-closure is solely a qualitative property, we introduce a quantitative performance index which characterizes the grasp robustness. This index corresponds to the maximum offset of the object's position $\delta_{x}$ for which the grasp is form-closed (Fig. 6). It allows the system to overcome UAVs positioning errors.

Here, the pulley position is an input value deduced from the optimization made in Sec. 3. Concerning the grasped object, we consider two circles with different diameters $(200 \mathrm{~mm}$ and $300 \mathrm{~mm}$ ). The parameters to optimize completely define the geometry of the gripper. They are presented in Fig. 6 .

In order to find the best values of these design parameters, we define a large range of values for each of them and test all potential combinations. For each set of parameters, $\delta_{x}$ is increased incrementally from 0 to a maximum value $\delta_{x \max }$ that still guarantees form-closure of the grasp. The following design constraints 


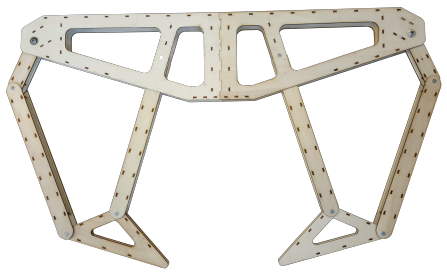

Fig. 7: Prototype made in plywood

must be verified: no collision between distal phalanges, no collision between the actuating bar and the quadrotors and that the bars $b_{1}$ and $c_{1}$ should not be parallel in order to avoid singular configurations (Fig. 6). The best set of parameters is thus the one for which $\delta_{x \max }$ is maximum. Final results are shown in Tab. 2.

\begin{tabular}{c|c|c|c|c|c|c|c|c|c} 
Parameters & $a[\mathrm{~mm}]$ & $b[\mathrm{~mm}]$ & $l_{1}[\mathrm{~mm}]$ & $l_{2}[\mathrm{~mm}]$ & $a_{1}[\mathrm{~mm}]$ & $c_{1}[\mathrm{~mm}]$ & $b_{1}[\mathrm{~mm}]$ & $\psi\left[^{\circ}\right]$ & $\delta_{\text {xmax }}[\mathrm{mm}]$ \\
\hline $\begin{array}{c}\text { Optimal } \\
\text { values }\end{array}$ & 240 & 150 & 536 & 214 & 624 & 80 & 530 & 55 & 65
\end{tabular}

Table 2: Result of form-closure optimization

A first prototype of the planar mechanism has been realized in $5 \mathrm{~mm}$ poplar plywood with a laser-cutting machine (Trotec Speedy 300) (Fig. 7). The finger's weight is about $1 \mathrm{~kg}$ and should be further reduced in order to increase the payload. This prototype is a first step before machining a 3D manipulator and it will be used to test grasp planning and control algorithms.

\section{Conclusion and future work}

This paper presents a new flying manipulator equipped with four underactuated fingers and actuated by four quadrotors. An optimization method is proposed to determine the position and orientation of quadrotors for a two-finger planar mechanism in order to maximize its manipulability. A second optimization method is introduced to determine the geometric parameters of the underactuated finger, so as to maximize the capability of the gripper to produce first-order form-closed grasps and its robustness to positioning error of the UAV relative to the object. A first prototype of a planar mechanism was then presented.

Further works will consist in modeling the dynamics of the system and controlling it during the successive flying tasks (before contacting the object, while grasping and manipulation in free space). A study on the grasp planning for different shapes of object is contemplated too. 


\section{Acknowledgement}

We would like to thank the Icam for its financial support in the prototype manufacture.

\section{References}

1. Birglen, L., Gosselin, C.: Optimal design of 2-phalanx underactuated fingers. In: International Conference on Intelligent Manipulation and Grasping (2004)

2. Gioioso, G., Franchi, A., Salvietti, G., Scheggi, S., Prattichizzo, D.: The flying hand: A formation of UAVs for cooperative aerial tele-manipulation. In: IEEE International Conference on Robotics and Automation (ICRA) (2014)

3. Goodarzi, F., Lee, T.: Stabilization of a rigid body payload with multiple cooperative quadrotors. ASME Journal of Dynamic Systems, Measurement and Control 138(12), 121,001 (2016)

4. Kondak, K., Huber, F., Schwarzbach, M., Laiacker, M., Sommer, D., Bejar, M., Ollero, A.: Aerial manipulation robot composed of an autonomous helicopter and a 7 degrees of freedom industrial manipulator. In: IEEE International Conference on Robotics and Automation (2014)

5. Krut, S., Bégoc, V., Dombre, E., Pierrot, F.: Extension of the form-closure property to underactuated hands. IEEE Transactions on Robotics (5), 853-866 (2010)

6. Krut, S., Company, O., Pierrot, F.: Force performance indexes for parallel mechanisms with actuation redundancy, especially for parallel wire-driven manipulators. In: IEEE/RSJ International Conference on Intelligent Robots and Systems (IROS) (2004)

7. Lee, T.: Geometric control of multiple quadrotor UAVs transporting a cablesuspended rigid body. In: IEEE 53rd Annual Conference on Decision and Control (CDC) (2014)

8. Manubens, M., Devaurs, D., Ros, L., Cortès, J.: A motion planning approach to 6-d manipulation with aerial towed-cable systems. In: International Micro Air Vehicle Conference and Flight Competition (2013)

9. Mellinger, D., Shomin, M., Michael, N., Kumar, V.: Cooperative grasping and transport using multiple quadrotors. In: Distributed Autonomous Robotic Systems, Springer Tracts in Advanced Robotics, vol. 83, pp. 545-558 (2013)

10. Orsag, M., Korpela, C., Oh, P.: Modeling and control of MM-UAV: Mobile manipulating unmanned aerial vehicle. Journal of Intelligent \& Robotic Systems 69(1), 227-240 (2013)

11. Pounds, P., Bersak, D., M. Dollar, A.: Grasping from the air: Hovering capture and load stability. In: IEEE International Conference on Robotics and Automation (2011)

12. Six, D., Chriette, A., Briot, S., Martinet, P.: Dynamic modeling and trajectory tracking controller of a novel flying parallel robot. In: the 20th IFAC World Congress (2017)

13. Thomas, J., Polin, J., Sreenath, K., Kumar, V.: Avian-inspired grasping for quadrotor micro UAVs. In: ASME 2013 International Design Engineering Technical Conferences and Computers and Information in Engineering Conference (2013)

14. Welch, C., Akbarisamani, S., Detweiler, C., Nelson, C.: Lightweight, low-cost, compliant grasper for UAV-based pick-and-place operations. In: ASME International Design Engineering Technical Conferences and Computers and Information in Engineering Conference (2015) 